\title{
Ground state and nodal solutions for a class of double phase problems
}

\author{
N.S. Papageorgiou, V.D. Rădulescuł, D.D. Repovš ${ }^{\ddagger}$
}

May 25, 2020

\begin{abstract}
We consider a double phase problem driven by the sum of the $p$-Laplace operator and a weighted $q$-Laplacian $(q<p)$, with a weight function which is not bounded away from zero. The reaction term is $(p-1)$-superlinear. Employing the Nehari method, we show that the equation has a ground state solution of constant sign and a nodal (sign-changing) solution.
\end{abstract}

2010 AMS Subject Classification: 35J60, 35D05.

Keywords: Double phase operator, weight function, superlinear reaction, Nehari manifold, ground state solution, nodal solution.

\section{Introduction}

This paper was motivated by several recent contributions to the qualitative analysis of nonlinear problems with unbalanced growth. We mainly refer to the pioneering contributions of Marcellini $[18,19,20]$ who studied lower semicontinuity and regularity properties of minimizers of certain quasiconvex integrals. Problems of this type arise in nonlinear elasticity and are connected with the deformation of an elastic body, cf. Ball $[1,2]$.

\subsection{Unbalanced problems and their historical traces}

Let $\Omega$ be a bounded domain in $\mathbb{R}^{N}(N \geq 2)$ with a smooth boundary. If $u: \Omega \rightarrow \mathbb{R}^{N}$ is the displacement and $D u$ is the $N \times N$ matrix of the deformation gradient, then the total energy can be represented by an integral of the type

$$
I(u)=\int_{\Omega} f(x, D u(x)) d x
$$

where the energy function $f=f(x, \xi): \Omega \times \mathbb{R}^{N \times N} \rightarrow \mathbb{R}$ is quasiconvex with respect to $\xi$, see Morrey [21]. One of the simplest examples considered by Ball is given by functions $f$ of the type

$$
f(\xi)=g(\xi)+h(\operatorname{det} \xi)
$$

where $\operatorname{det} \xi$ is the determinant of the $N \times N$ matrix $\xi$, and $g, h$ are nonnegative convex functions, which satisfy the growth conditions

$$
g(\xi) \geq c_{1}|\xi|^{p} ; \quad \lim _{t \rightarrow+\infty} h(t)=+\infty,
$$

where $c_{1}$ is a positive constant and $1<p<N$. The condition $p \leq N$ is necessary to study the existence of equilibrium solutions with cavities, that is, minima of the integral (1) that are discontinuous at one point

\footnotetext{
* National Technical University, Zografou Campus, 15780 Athens, Greece \& Institute of Mathematics, Physics and Mechanics, 1000 Ljubljana, Slovenia.

${ }^{\dagger}$ AGH University of Science and Technology, 30-059 Krakow, Poland \& Institute of Mathematics, Physics and Mechanics, 1000 Ljubljana, Slovenia.

${ }_{\ddagger}^{\ddagger}$ Faculty of Education and Faculty of Mathematics and Physics, University of Ljubljana \& Institute of Mathematics, Physics and Mechanics, 1000 Ljubljana, Slovenia. E-mail: dusan.repovs@guest.arnes.si
} 
where a cavity forms; in fact, every $u$ with finite energy belongs to the Sobolev space $W^{1, p}\left(\Omega, \mathbb{R}^{N}\right)$, and thus it is a continuous function if $p>N$. In accordance with these problems arising in nonlinear elasticity, Marcellini $[18,19]$ considered continuous functions $f=f(x, u)$ with unbalanced growth that satisfy

$$
c_{1}|u|^{p} \leq|f(x, u)| \leq c_{2}\left(1+|u|^{q}\right) \quad \text { for all }(x, u) \in \Omega \times \mathbb{R}
$$

where $c_{1}, c_{2}$ are positive constants and $1 \leq p \leq q$. Regularity and existence of solutions of elliptic equations with $p, q$-growth conditions were studied in [19].

The study of non-autonomous functionals characterized by the fact that the energy density changes its ellipticity and growth properties according to the point has been continued in a series of remarkable papers by Mingione et al. [3, 4, 10, 11]. These contributions are in relationship with the work of Zhikov [32, 33], which describe the behavior of phenomena arising in nonlinear elasticity. In fact, Zhikov intended to provide models for strongly anisotropic materials in the context of homogenisation. In particular, he considered the following model functional

$$
\mathcal{P}_{p, q}(u):=\int_{\Omega}\left(|D u|^{p}+a(x)|D u|^{q}\right) d x, \quad 0 \leq a(x) \leq L, 1<p<q,
$$

where the modulating coefficient $a(x)$ dictates the geometry of the composite made of two differential materials, with hardening exponents $p$ and $q$, respectively.

Another significant model example of a functional with $(p, q)$-growth studied by Mingione et al. is given by

$$
u \mapsto \int_{\Omega}|D u|^{p} \log (1+|D u|) d x, \quad p \geq 1,
$$

which is a logarithmic perturbation of the $p$-Dirichlet energy.

\section{$1.2 \quad$ Statement of the problem}

Let $\Omega \subseteq \mathbb{R}^{N}$ be a bounded domain with a Lipschitz boundary $\partial \Omega$. In this paper we study the following double phase Dirichlet problem

$$
-\Delta_{p} u-\operatorname{div}\left(a(z)|D u|^{q-2} D u\right)=f(z, u) \text { in } \Omega,\left.u\right|_{\partial \Omega}=0,1<q<p .
$$

In this problem, $\Delta_{p}$ denotes the $p$-Laplace differential operator defined by

$$
\Delta_{p} u=\operatorname{div}\left(|D u|^{p-2} D u\right) \text { for all } u \in W_{0}^{1, p}(\Omega) .
$$

So, in problem (3) the differential operator is the sum of a $p$-Laplacian and of a weighted $q$-Laplace operator with $q<p$ and weight $a \in L^{\infty}(\Omega), a(z)>0$ for a.a. $z \in \Omega$. The integrand in the energy functional corresponding to this differential operator is $k(z, t)=\frac{1}{p} t^{p}+\frac{1}{q} a(z) t^{q}$ for all $t \geq 0$. This integrand exhibits balanced growth since we have for some $c_{0}>0$

$$
\frac{1}{p} t^{p} \leq k(z, t) \leq c_{0}\left(1+t^{p}\right) \text { for a.a. } z \in \Omega, \text { and all } t>0 .
$$

However, the presence of the weight $a(\cdot)$ which is not continuous and is not bounded away from zero, does not permit the use of the nonlinear regularity theory of Lieberman [16] and the nonlinear strong maximum principle of Pucci and Serrin [29, pp. 111,120]. In fact, these were the main tools used in the analysis of $(p, q)$ equations (that is, equations driven by the sum of a $p$-Laplacian and of a $q$-Laplacian with no weight) and they led to multiplicity results for such equations. We refer to the works of Mugnai and Papageorgiou [22], Papageorgiou and Rădulescu [23], and Papageorgiou, Vetro and Vetro [27], which deal with $(p, q)$-equations with a $(p-1)$-superlinear reaction term. In problem (3) the reaction (source) term $f(z, x)$ is a measurable function which is $C^{1}$ in the $x$-variable and $(p-1)$-superlinear as $x \rightarrow \pm \infty$. The approach developed in the present paper is based on the Nehari method.

We mention that double phase equations arise in mathematical models of various physical phenomena. We mention the works of Benci, D'Avenia, Fortunato and Pisani [5] (in quantum physics), Cherfils and 
Ilyasov [6] (in reaction-diffusion systems), Zhikov [32, 33] (in nonlinear elasticity theory). Recently, in a series of remarkable papers Mingione et al. (see $[3,4,10,11]$ ) produced local regularity results for equations driven by the unbalanced double phase operator, namely when

$$
-\operatorname{div}\left(a(z)|D u|^{p-2} D u\right)-\Delta_{q} u \text { with } 1<q<p .
$$

In this case the integrand in the corresponding energy functional is

$$
\hat{k}(z, x)=\frac{1}{p} a(z) t^{p}+\frac{1}{q} t^{q} \text { for all } t>0
$$

and we have

$$
\frac{1}{q} t^{q} \leq \hat{k}(z, x) \leq \hat{c}_{0}\left(1+t^{p}\right) \text { for a.a. } z \in \Omega, \text { all } t>0, \text { some } \hat{c}_{0}>0 \text { (unbalanced growth). }
$$

For such equations the appropriate functional space framework is provided by Musielak-Orlicz-Sobolev spaces. This leads to more restrictive conditions on the weight $a(\cdot)$, which cannot be discontinuous and also there are restrictions on the exponents $1<q<p$, namely $\frac{p}{q}<1+\frac{1}{N}$. So, $p$ and $q$ cannot differ too much. We also refer to the works of Colasuonno and Squassina [9], Ge, Lv and Lu [13], Liu and Dai [17], Papageorgiou, Rădulescu and Repovš [24, 26], Qihu and Rădulescu [30], and the survey paper of Rădulescu [31].

\section{Mathematical preliminaries and hypotheses}

The balanced growth of the integrand $k(z, \cdot)$ permits the use of the Sobolev space $W_{0}^{1, p}(\Omega)$ for the study of problem (3). By $\|\cdot\|$ we denote the norm of $W_{0}^{1, p}(\Omega)$. Using the Poincaré inequality, we have

$$
\|u\|=\|D u\|_{p} \text { for all } u \in W_{0}^{1, p}(\Omega) .
$$

Consider the following nonlinear eigenvalue problem

$$
-\Delta_{p} u=\hat{\lambda}|u|^{p-2} u \text { in } \Omega,\left.u\right|_{\partial \Omega}=0 .
$$

It is well-known that this eigenvalue problem has a smallest eigenvalue $\hat{\lambda}_{1}$, which is isolated, simple and the corresponding eigenfunctions have fixed sign. By $\hat{u}_{1}$ we denote the positive, $L^{p}$-normalized (that is, $\left.\left\|\hat{u}_{1}\right\|_{p}=1\right)$ eigenfunction. Then $\hat{u}_{1} \in C_{0}^{1}(\bar{\Omega}), \hat{u}_{1}(z)>0$ for all $z \in \Omega$ and $\left.\frac{\partial \hat{u}_{1}}{\partial n}\right|_{\partial \Omega}<0$, with $n(\cdot)$ being the outward unit normal on $\partial \Omega$. We have

$$
\hat{\lambda}_{1}=\inf \left\{\frac{\|D u\|_{p}^{p}}{\|u\|_{p}^{p}}: u \in W_{0}^{1, p}(\Omega), u \neq 0\right\}, \quad\left\|D \hat{u}_{1}\right\|_{p}^{p}=\hat{\lambda}_{1}\left\|\hat{u}_{1}\right\|_{p}^{p}
$$

For details we refer to Gasinski and Papageorgiou [12].

We introduce the conditions on the weight $a(\cdot)$.

$H(a): a \in L^{\infty}(\Omega), a(z)>0$ for a.a. $z \in \Omega$.

We define the following quantity related to the double phase differential operator:

$$
\vartheta=\inf \left\{\frac{\|D u\|_{p}^{p}+\frac{p}{q} \int_{\Omega} a(z)|D u|^{q} d z}{\|u\|_{p}^{p}}: u \in W_{0}^{1, p}(\Omega), u \neq 0\right\} .
$$

Lemma 2.1. If hypothesis $H(a)$ holds, then $\vartheta=\hat{\lambda}_{1}$.

Proof. By (4), (5) and hypothesis $H(a)$, it is clear that we have

$$
\hat{\lambda}_{1} \leq \vartheta
$$


On the other hand, for every $t>0$ we have

$$
\begin{aligned}
\vartheta & \leq \frac{\left\|D\left(t \hat{u}_{1}\right)\right\|_{p}^{p}+\frac{p}{q} \int_{\Omega} a(z)\left|D\left(t \hat{u}_{1}\right)\right|^{q} d z}{t^{p}} \quad\left(\text { recall that }\left\|\hat{u}_{1}\right\|_{p}=1\right) \\
& =\left\|D \hat{u}_{1}\right\|_{p}^{p}+\frac{1}{t^{p-q}} \frac{p}{q} \int_{\Omega} a(z)\left|D \hat{u}_{1}\right|^{q} d z \\
& =\hat{\lambda}_{1}+\frac{1}{t^{p-q}} \frac{p}{q} \int_{\Omega} a(z)\left|D \hat{u}_{1}\right|^{q} d z(\text { see }(4)) .
\end{aligned}
$$

We let $t \rightarrow+\infty$. Since $q<p$, we obtain

$$
\begin{aligned}
\vartheta & \leq \hat{\lambda}_{1} \\
\Rightarrow \vartheta & =\hat{\lambda}_{1}(\text { see }(6)) .
\end{aligned}
$$

The proof is now complete.

Next, we introduce the conditions on the reaction function $f(z, x)$. Recall that $p^{*}$ denotes the critical Sobolev exponent corresponding to $p$, which is defined by

$$
p^{*}= \begin{cases}\frac{N p}{N-p}, & \text { if } p<N \\ +\infty, & \text { if } N \leq p .\end{cases}
$$

$H(f): f: \Omega \times \mathbb{R} \rightarrow \mathbb{R}$ is a measurable function such that for a.a. $z \in \Omega, f(z, 0)=0, f(z, \cdot) \in C^{1}(\mathbb{R} \backslash\{0\})$ and

(i) $\left|f_{x}^{\prime}(z, x)\right| \leq a_{0}(z)\left(1+|x|^{r-2}\right)$ for a.a. $z \in \Omega$ and all $x \in \mathbb{R}$, with $a_{0} \in L^{\infty}(\Omega), p<r<p^{*}$;

(ii) if $F(z, x)=\int_{0}^{x} f(z, s) d s$, then $\lim _{x \rightarrow \pm \infty} \frac{F(z, x)}{|x|^{p}}=+\infty$ uniformly for a.a. $z \in \Omega$ and there exist $\tau \in$ $\left(\max \left\{1,(r-p) \frac{N}{p}\right\}, p^{*}\right)$ and $\beta_{0}>0$ such that

$$
\beta_{0} \leq \liminf _{x \rightarrow \pm \infty} \frac{f(z, x) x-p F(z, x)}{|x|^{\tau}} \text { uniformly fo a.a. } z \in \Omega
$$

(iii) $\lim _{x \rightarrow 0} \frac{f(z, x)}{|x|^{q-2} x}=0$ uniformly for a.a. $z \in \Omega$;

(iv) $0<(p-1) f(z, x) x \leq f_{x}^{\prime}(z, x) x^{2}$ for a.a. $z \in \Omega$ and all $x \neq 0$.

Remarks. Hypothesis $H(f)(i i)$ implies that for a.a. $z \in \Omega, f(z, \cdot)$ is $(p-1)$-superlinear. Hypothesis $H(f)(i v)$ implies that for a.a. $z \in \Omega$ we have

$$
\begin{gathered}
x \mapsto \frac{f(z, x)}{|x|^{p-1}} \text { is increasing on }(0, \infty) \text { and on }(-\infty, 0), \\
x \mapsto f(z, x) x-p F(z, x) \text { is increasing in }|x| .
\end{gathered}
$$

Note that the above monotonicities are not strict, contrary to what was used in [13, 17].

Examples. Consider the following functions (for the sake of simplicity, we drop the $z$-dependence):

$$
\begin{gathered}
f_{1}(x)=|x|^{r-2} x \text { with } p<r<p^{*} \\
f_{2}(x)=\left\{\begin{array}{ll}
|x|^{s-2} x-|x|^{p-2} x, & \text { if }|x| \leq 1 \\
k|x|^{p-2} x \ln |x|, & \text { if } 1<|x|
\end{array} \text { with } p<s, k=s-p>0 .\right.
\end{gathered}
$$

Note that $f_{2}(\cdot)$ although $(p-1)$-superlinear, does not satisfy the well known Ambrosetti-Rabinowitz condition, which is common in problems with a superlinear reaction (see [17]). 
Let $\varphi: W_{0}^{1, p}(\Omega) \rightarrow \mathbb{R}$ be the energy (Euler) functional for problem (3) defined by

$$
\varphi(u)=\frac{1}{p}\|D u\|_{p}^{p}+\frac{1}{q} \int_{\Omega} a(z)|D u|^{q} d z-\int_{\Omega} F(z, u) d z \text { for all } u \in W_{0}^{1, p}(\Omega) .
$$

Evidently, $\varphi \in C^{1}\left(W_{0}^{1, p}(\Omega)\right)$. We say that $u \in W_{0}^{1, p}(\Omega)$ is a "weak solution" of problem (3), if

$$
\left\langle A_{p}(u), h\right\rangle+\int_{\Omega} a(z)|D u|^{q-2}(D u, D h)_{\mathbb{R}^{N}} d z=\int_{\Omega} f(z, u) h d z \text { for all } h \in W_{0}^{1, p}(\Omega),
$$

with $A_{p}: W_{0}^{1, p}(\Omega) \rightarrow W^{-1, p^{\prime}}(\Omega)=W_{0}^{1, p}(\Omega)^{*}\left(\frac{1}{p}+\frac{1}{p^{\prime}}=1\right)$ being the nonlinear map defined by

$$
\left\langle A_{p}(u), h\right\rangle=\int_{\Omega}|D u|^{p-2}(D u, D h)_{\mathbb{R}^{N}} d z \text { for all } u, h \in W_{0}^{1, p}(\Omega) .
$$

As we have already mentioned, our approach is based on the Nehari method. For this reason, we introduce the Nehari manifold for $\varphi$, defined by

$$
\mathcal{N}=\left\{u \in W_{0}^{1, p}(\Omega):\left\langle\varphi^{\prime}(u), u\right\rangle=0, u \neq 0\right\} .
$$

As above, we denote by $\langle\cdot, \cdot\rangle$ the duality brackets for the pair $\left(W^{-1, p^{\prime}}(\Omega), W_{0}^{1, p}(\Omega)\right)$. Evidently, the nontrivial weak solutions of (3) belong to $\mathcal{N}$. Also, since we want to produce nodal solutions, we will use the following set

$$
\mathcal{N}_{0}=\left\{u \in W_{0}^{1, p}(\Omega): u^{+} \in \mathcal{N},-u^{-} \in \mathcal{N}\right\} .
$$

Recall that $u^{+}=\max \{u, 0\}, u^{-}=\max \{-u, 0\}$ for all $u \in W_{0}^{1, p}(\Omega)$. We have $u^{+}, u^{-} \in W_{0}^{1, p}(\Omega)$ and $u=u^{+}-u^{-},|u|=u^{+}+u^{-}$. In the sequel, we will say "solution" and mean "weak solution". Also, by $|\cdot|_{N}$ we denote the Lebesgue measure on $\mathbb{R}^{N}$.

\section{Ground state solutions}

In this section we prove the existence of a solution of (3) which minimizes $\left.\varphi\right|_{N}$. Such a solution is known as a "ground state solution".

Proposition 3.1. If hypotheses $H(a), H(f)$ hold and $u \in W_{0}^{1, p}(\Omega), u \neq 0$, then there exists a unique $t_{u}>0$ such that $t_{u} u \in \mathcal{N}$.

Proof. We consider the fibering map $\mu_{u}:(0, \infty) \rightarrow \mathbb{R}$ defined by

$$
\mu_{u}(t)=\varphi(t u) \text { for all } t>0 .
$$

Evidently, $\mu_{u} \in C^{1}(0, \infty)$ and using the chain rule, we have

$$
\mu_{u}^{\prime}(t)=t^{p-1}\|D u\|_{p}^{p}+t^{q-1} \int_{\Omega} a(z)|D u|^{q} d z-\int_{\Omega} f(z, t u) u d z .
$$

We see that

$$
\mu_{u}^{\prime}(t)=0 \Leftrightarrow t u \in \mathcal{N} .
$$

So, we consider the equation $\mu_{u}^{\prime}(t)=0$. This is equivalent to

$$
\|D u\|_{p}^{p}=\int_{\Omega} \frac{f(z, t u)}{t^{p-1}} u d z-\frac{1}{t^{p-q}} \int_{\Omega} a(z)|D u|^{q} d z .
$$

In relation (7), the right-hand side is strictly increasing (see hypothesis $H(f)(i)$ and recall that $q<p$ ). So, there exists a unique $t_{u}>0$ such that

$$
\begin{aligned}
& \mu_{u}^{\prime}\left(t_{u}\right)=0 \\
\Rightarrow & \left\langle\varphi^{\prime}\left(t_{u} u\right), u\right\rangle=0 \text { (by the chain rule), } \\
\Rightarrow & \left\langle\varphi^{\prime}\left(t_{u} u\right), t_{u} u\right\rangle=0 \\
\Rightarrow & t_{u} u \in \mathcal{N} .
\end{aligned}
$$

The proof is complete. 
Corollary 3.2. If hypotheses $H(a), H(f)$ hold, then $\mathcal{N} \neq \emptyset$.

Proposition 3.3. If hypotheses $H(a), H(f)$ hold and $u \in \mathcal{N}$, then $\varphi(t u) \leq \varphi(u)$ for all $t>0$.

Proof. We consider the fibering map $\mu_{u}(\cdot)$ introduced in the proof of Proposition 3.1. Since $u \in \mathcal{N}$, we have $\mu_{u}^{\prime}(1)=0$ and this is the unique critical point of $\mu_{u}(\cdot)$.

On account of hypotheses $H(f)(i),(i i)$, given $\eta>0$, we can find $c_{\eta}>0$ such that

$$
F(z, x) \geq \frac{\eta}{p}|x|^{p}-c_{\eta} \text { for a.a. } z \in \Omega \text { and all } x \in \mathbb{R} .
$$

Then for any $t>0$ we have

$$
\begin{aligned}
\varphi(t u) & \leq \frac{t^{p}}{p}\|D u\|_{p}^{p}+\frac{t^{q}}{q} \int_{\Omega} a(z)|D u|^{q} d z-\frac{t^{p}}{p} \eta\|u\|_{p}^{p}+c_{\eta}|\Omega|_{N} \text { (see (8)) } \\
& =\frac{t^{p}}{p}\left[\|D u\|_{p}^{p}-\eta\|u\|_{p}^{p}\right]+\frac{t^{q}}{q} \int_{\Omega} a(z)|D u|^{q} d z+c_{\eta}|\Omega|_{N} .
\end{aligned}
$$

Choosing $\eta>0$ such that $\|D u\|_{p}^{p}<\eta\|u\|_{p}^{p}$, we have

$$
\varphi(t u) \leq c_{1} t^{q}-c_{2} t^{p}+c_{\eta}|\Omega|_{N} \text { for some } c_{1}>0, c_{2}>0 .
$$

Since $q<p$, from (9) we see that

$$
\mu_{u}(t)=\varphi(t u)<0 \text { for } t>1 \text { big enough. }
$$

On the other hand, hypotheses $H(f)(i),($ iii $)$ imply that given $\varepsilon>0$, we can find $c_{\varepsilon}>0$ such that

$$
F(z, x) \leq \frac{\varepsilon}{q}|x|^{q}+c_{\varepsilon}|x|^{r} \text { for a.a. } z \in \Omega \text { and all } x \in \mathbb{R} .
$$

Therefore for $t>0$ we have

$$
\begin{aligned}
\varphi(t u) & \geq \frac{t^{p}}{p}\|D u\|_{p}^{p}+\frac{t^{q}}{q} \int_{\Omega} a(z)|D u|^{q} d z-\frac{\varepsilon t^{q}}{q}\|u\|_{q}^{q}-c_{\varepsilon} t^{r}\|u\|_{r}^{r} \text { (see (11)) } \\
& \geq \frac{t^{p}}{p}\|u\|^{p}+\frac{t^{q}}{q}\left[\int_{\Omega} a(z)|D u|^{q} d z-\varepsilon\|u\|_{q}^{q}\right]-c_{3} t^{r}\|u\|^{r} \text { for some } c_{3}>0 .
\end{aligned}
$$

Since $\int_{\Omega} a(z)|D u|^{q} d z>0$ (see hypothesis $H(a)$ and recall that $u \neq 0$ ), by choosing $\varepsilon>0$ small enough, we have $\int_{\Omega} a(z)|D u|^{q} d z \geq \varepsilon\|u\|_{q}^{q}$ and so

$$
\varphi(t u) \geq c_{4} t^{p}-c_{5} t^{r} \text { for some } c_{4}, c_{5}>0 .
$$

Since $p<r$, it follows that

$$
\mu_{u}(t)=\varphi(t u)>0 \text { for all } t \in(0,1) \text { small enough. }
$$

From (10) and (12) we can infer that $t_{u}=1$ is a maximizer of $\mu_{u}(\cdot)$ and so we have $\varphi(t u) \leq \varphi(u)$ for all $t>0$.

Let $m=\inf _{\mathcal{N}} \varphi$.

Proposition 3.4. If hypotheses $H(a), H(f)$ hold, then $m>0$.

Proof. Let $u \in W_{0}^{1, p}(\Omega) \backslash\{0\}$. We have

$$
\varphi(u) \geq \frac{1}{p}\|D u\|_{p}^{p}+\frac{1}{q}\left[\int_{\Omega} a(z)|D u|^{q} d z-\varepsilon\|u\|_{q}^{q}\right]-c_{6}\|u\|^{r} \text { for some } c_{6}>0 \text { (see (11)). }
$$


Let $c_{7} \in\left(0, \frac{1}{p}\right)$. Since $q<p$, for $u \in W_{0}^{1, p}(\Omega)$ with $0<\|u\|_{p} \leq 1$, we have

$$
\begin{aligned}
\varphi(u) & \geq\left(\frac{1}{p}-c_{7}\right)\|D u\|_{p}^{p}+c_{7}\left[\|D u\|_{p}^{p}+\frac{1}{q c_{7}} \int_{\Omega} a(z)|D u|^{q} d z-\varepsilon c_{8}\|u\|_{p}^{p}\right]-c_{6}\|u\|^{r} \text { for some } c_{8}>0 \\
& =\left(\frac{1}{p}-c_{7}\right)\|D u\|_{p}^{p}+c_{7}\left[\|D u\|_{p}^{p}+\frac{p}{q} \int_{\Omega} \frac{a(z)}{c_{9}}|D u|^{q} d z-\varepsilon c_{8}\|u\|_{p}^{p}\right]-c_{6}\|u\|^{r} \text { with } c_{9}=\frac{1}{p c_{7}}>0 \\
& \geq\left(\frac{1}{p}-c_{7}\right)\|D u\|_{p}^{p}+c_{7}\left(\hat{\lambda}-\varepsilon c_{8}\right)\|u\|_{p}^{p}-c_{6}\|u\|^{r} \text { (see Lemma 2.1). }
\end{aligned}
$$

Choosing $\varepsilon \in\left(0, \hat{\lambda}_{1} / c_{8}\right)$, we obtain

$$
\begin{aligned}
\varphi(u) \geq & c_{10}\|u\|^{p}-c_{6}\|u\|^{r} \\
& \text { for some } c_{10}>0 \text { and all } u \in W_{0}^{1, p}(\Omega) \text { with }\|u\|_{p} \leq 1 .
\end{aligned}
$$

Because $p<r$, we can find $\rho \in(0,1)$ small, $\rho \leq \frac{1}{\hat{\lambda}_{1}}$ such that

$$
\left.\varphi(u) \geq \eta_{0}>0 \text { for all }\|u\|=\rho \text { (note that }\|u\|_{p} \leq \frac{1}{\hat{\lambda}_{1}}\|u\| \leq \rho\right) .
$$

Now we consider $u \in \mathcal{N}$ and choose $\tau_{u}>0$ such that $\tau_{u}\|u\|=\rho$. Then by Proposition 3.3, we have

$$
\begin{aligned}
& \varphi(u) \geq \varphi\left(\tau_{u} u\right) \geq \eta_{0}>0, \\
\Rightarrow \quad & m=\inf _{\mathcal{N}} \varphi>0 .
\end{aligned}
$$

The proof is now complete.

The Nehari manifold is much smaller than $W_{0}^{1, p}(\Omega)$ and so some properties of $\varphi$ which evidently fail globally, can be true for $\left.\varphi\right|_{\mathcal{N}}$. This is illustrated in the next proposition.

Proposition 3.5. If hypotheses $H(a), H(f)$ hold, then $\left.\varphi\right|_{\mathcal{N}}$ is coercive.

Proof. Evidently, it suffices to show that if $\left\{u_{n}\right\}_{n \geq 1} \subseteq \mathcal{N}$ and $\varphi\left(u_{n}\right) \leq M$ for some $M>0$ and all $n \in \mathbb{N}$, then $\left\{u_{n}\right\}_{n \geq 1} \subseteq W_{0}^{1, p}(\Omega)$ is bounded.

We have

$$
\left\|D u_{n}\right\|_{p}^{p}+\frac{p}{q} \int_{\Omega} a(z)\left|D u_{n}\right|^{q} d z-\int_{\Omega} p F\left(z, u_{n}\right) d z \leq p M \text { for all } n \in \mathbb{N} .
$$

Since $u_{n} \in \mathcal{N}$, we have

$$
\begin{aligned}
& \left\langle\varphi^{\prime}\left(u_{n}\right), u_{n}\right\rangle=0 \text { for all } n \in \mathbb{N}, \\
\Rightarrow \quad & -\left\|D u_{n}\right\|_{p}^{p}-\int_{\Omega} a(z)\left|D u_{n}\right|^{q} d z+\int_{\Omega} f\left(z, u_{n}\right) u_{n} d z=0 \text { for all } n \in \mathbb{N} .
\end{aligned}
$$

We add (13) and (14) and have

$$
\begin{aligned}
& \left(\frac{p}{q}-1\right) \int_{\Omega} a(z)\left|D u_{n}\right|^{q} d z+\int_{\Omega}\left[f\left(z, u_{n}\right) u_{n}-p F\left(z, u_{n}\right)\right] d z \leq p M \text { for all } n \in \mathbb{N}, \\
\Rightarrow & \int_{\Omega}\left[f\left(z, u_{n}\right) u_{n}-p F\left(z, u_{n}\right)\right] d z \leq p M \text { for all } n \in \mathbb{N}
\end{aligned}
$$

(recall that $q<p$ and see hypothesis $H(a)$ ).

Hypotheses $H(f)(i),(i i)$ imply that given $\beta_{1} \in\left(0, \beta_{0}\right)$, we can find $c_{11}=c_{11}\left(\beta_{1}\right)>0$ such that

$$
\beta_{1}|x|^{\tau}-c_{11} \leq f(z, x) x-p F(z, x) \text { for a.a. } z \in \Omega \text { and all } x \in \mathbb{R} .
$$

Using (16) in (15), we obtain that

$$
\left\{u_{n}\right\}_{n \geq 1} \subseteq L^{\tau}(\Omega) \text { is bounded. }
$$


We first assume that $N \neq p$. It is clear by hypothesis $H(f)(i i)$ that without any loss of generality, we may assume that $\tau<r<p^{*}$. Let $t \in(0,1)$ such that

$$
\frac{1}{r}=\frac{1-t}{\tau}+\frac{t}{p^{*}}
$$

The interpolation inequality (see for example Papageorgiou and Winkert [28, p.116]) implies that

$$
\begin{aligned}
\left\|u_{n}\right\|_{r} \leq & \left\|u_{n}\right\|_{\tau^{1}}^{1-t}\left\|u_{n}\right\|_{p^{*}}^{t} \text { for all } n \in \mathbb{N}, \\
\Rightarrow\left\|u_{n}\right\|_{r}^{r} \leq & c_{12}\left\|u_{n}\right\|^{t r} \text { for some } c_{12}>0 \text { and all } n \in \mathbb{N} \\
& \quad \text { (see (17) and use the Sobolev embedding theorem). }
\end{aligned}
$$

Hypothesis $H(f)(i)$ implies that

$$
f(z, x) x \leq c_{13}\left(1+|x|^{r}\right) \text { for a.a. } z \in \Omega, \text { all } x \in \mathbb{R} \text { and some } c_{13}>0 .
$$

From (14) we have

$$
\begin{aligned}
\left\|D u_{n}\right\|_{p}^{p}+\int_{\Omega} a(z)\left|D u_{n}\right|^{q} d z & =\int_{\Omega} f\left(z, u_{n}\right) u_{n} d z \\
& \leq c_{14}\left(1+\left\|u_{n}\right\|_{r}^{r}\right) \text { for some } c_{14}>0 \text { and all } n \in \mathbb{N} \text { (see (20)) } \\
& \leq c_{15}\left(1+\left\|u_{n}\right\|^{t r}\right) \text { for some } c_{15}>0 \text { and all } n \in \mathbb{N} \text { (see (19)), } \\
\Rightarrow\left\|u_{n}\right\|^{p} \leq c_{15}\left(1+\left\|u_{n}\right\|^{t r}\right) & \text { for all } n \in \mathbb{N} \text { (see hypothesis } H(a)) .
\end{aligned}
$$

From (18) and the condition on $\tau>1$ (see hypothesis $H(f)(i i)$ ), we see that $t r<p$. So, from (21) we can infer that

$$
\left\{u_{n}\right\}_{n \geq 1} \subseteq W_{0}^{1, p}(\Omega) \text { is bounded. }
$$

Next, assume that $N=p$. In this case $p^{*}=+\infty$, but by the Sobolev embedding theorem we have

$$
W_{0}^{1, p}(\Omega) \hookrightarrow L^{s}(\Omega) \text { for all } 1 \leq s<\infty .
$$

So, for the previous argument to work, we need to replace $p^{*}(=+\infty)$, with $s>r$ so big that

$$
\operatorname{tr}=\frac{s(r-\tau)}{s-\tau}<p(\text { see }(18) \text { and note that } N=p \Rightarrow r-p<\tau) .
$$

With such a choice of $s>r$, the previous argument works and we again reach (22). Therefore we can conclude that $\left.\varphi\right|_{\mathcal{N}}$ is coercive.

Proposition 3.6. If hypotheses $H(a), H(f)$ hold, then we can find $\hat{u} \in \mathcal{N}$ such that $\varphi(\hat{u})=m=\inf _{\mathcal{N}} \varphi>0$.

Proof. Let $\left\{u_{n}\right\}_{n \geq 1} \subseteq \mathcal{N}$ be a minimizing sequence, that is,

$$
\varphi\left(u_{n}\right) \downarrow m \text { as } n \rightarrow \infty .
$$

From Proposition 3.5, we have that

$$
\left\{u_{n}\right\}_{n \geq 1} \subseteq W_{0}^{1, p}(\Omega) \text { is bounded. }
$$

So, we may assume that

$$
u_{n} \stackrel{w}{\longrightarrow} \hat{u} \text { in } W_{0}^{1, p}(\Omega) \text { and } u_{n} \rightarrow \hat{u} \text { in } L^{r}(\Omega) .
$$

Since $u_{n} \in \mathcal{N}$, we have

$$
\left\|D u_{n}\right\|_{p}^{p}+\int_{\Omega} a(z)\left|D u_{n}\right|^{q} d z=\int_{\Omega} f\left(z, u_{n}\right) u_{n} d z \text { for all } n \in \mathbb{N} .
$$


In (24) we pass to the limit as $n \rightarrow \infty$ and use (23) and the weak lower semicontinuity of the norm functional. We obtain

$$
\|D \hat{u}\|_{p}^{p}+\int_{\Omega} a(z)|D \hat{u}|^{q} d z \leq \int_{\Omega} f(z, \hat{u}) \hat{u} d z .
$$

If $\hat{u}=0$, then from (23) and (24), we see that

$$
\begin{aligned}
& u_{n} \rightarrow 0 \text { in } W_{0}^{1, p}(\Omega), \\
\Rightarrow \quad & \varphi\left(u_{n}\right) \rightarrow m=\varphi(0)=0,
\end{aligned}
$$

a contradiction to Proposition 3.4. Therefore $\hat{u} \neq 0$.

If in $(25)$ we have equality, then $\hat{u} \in \mathcal{N}$ and $\varphi(\hat{u})=m$.

So, suppose that

$$
\|D \hat{u}\|_{p}^{p}+\int_{\Omega} a(z)|D \hat{u}|^{q} d z<\int_{\Omega} f(z, \hat{u}) \hat{u} d z .
$$

Using the fibering map $\mu_{\hat{u}}(\cdot)$ from the proof of Proposition 3.3 and from (26) we infer that

$$
\begin{aligned}
& \mu_{\hat{u}}(1)<0, \\
\Rightarrow \quad & \hat{t}=t_{\hat{u}} \in(0,1)(\text { see }(12) \text { and Proposition 3.1). }
\end{aligned}
$$

We have

$$
\begin{aligned}
m \leq & \varphi(\hat{t} \hat{u}) \\
= & \frac{1}{p}\|D(\hat{t} \hat{u})\|_{p}^{p}+\frac{1}{q} \int_{\Omega} a(z)|D(t \hat{u})|^{q} d z-\int_{\Omega} F(z, \hat{t} \hat{u}) d z \\
= & \frac{1}{p}\left[\int_{\Omega} f(z, \hat{t} \hat{u})(\hat{t} \hat{u}) d z-\int_{\Omega} a(z)|D(t \hat{u})|^{q} d z\right] \\
+ & \frac{1}{q} \int_{\Omega} a(z)|D(\hat{t} \hat{u})|^{q} d z-\int_{\Omega} F(z, \hat{t} \hat{u}) d z(\text { since } \hat{t} \hat{u} \in \mathcal{N}) \\
= & \int_{\Omega}\left[\frac{1}{p} f(z, t \hat{u})(t \hat{u})-F(z, \hat{t} \hat{u})\right] d z+\left(\frac{1}{q}-\frac{1}{p}\right) \int_{\Omega} a(z)|D \hat{u}|^{q} d z \\
< & \int_{\Omega}\left[\frac{1}{p} f(z, \hat{u}) \hat{u}-F(z, \hat{u})\right] d z+\left(\frac{1}{q}-\frac{1}{p}\right) \int_{\Omega} a(z)|D \hat{u}|^{q} d z \\
& (\operatorname{see}(27), \text { hypothesis } H(f)(i v) \text { and recall that } q<p) \\
\leq & \liminf _{n \rightarrow \infty}\left[\int_{\Omega}\left[\frac{1}{p} f\left(z, u_{n}\right) u_{n}-p F\left(z, u_{n}\right)\right] d z+\left(\frac{1}{q}-\frac{1}{p}\right) \int_{\Omega} a(z)\left|D u_{n}\right|^{q} d z\right] \\
& (\operatorname{see}(23) \text { and use Lemma } 1.12 \text { of Heinonen, Kilpeläinen and Martio }[14, \text { p.16]) } \\
= & m,
\end{aligned}
$$

a contradiction. Therefore we conclude that $\hat{u} \in \mathcal{N}$ and $\varphi(\hat{u})=m$.

The next proposition shows that the Nehari manifold is a natural constraint for $\hat{u}$ (see Papageorgiou, Rădulescu and Repovš [25, p.425]). In what follows, we denote

$$
K_{\varphi}=\left\{u \in W_{0}^{1, p}(\Omega): \varphi^{\prime}(u)=0\right\} \text { (the critical set of } \varphi \text { ). }
$$

Proposition 3.7. If hypotheses $H(a), H(f)$ hold and $\hat{u} \in \mathcal{N}$ satisfies $\varphi(\hat{u})=m$, then $\hat{u} \in K_{\varphi}$, $\hat{u}$ is a solution of $(3), \hat{u} \in W_{0}^{1, p}(\Omega) \cap L^{\infty}(\Omega)$ and $\hat{u}$ does not change sign.

Proof. Consider the function $k: W_{0}^{1, p}(\Omega) \rightarrow \mathbb{R}$ defined by

$$
k(u)=\|D u\|_{p}^{p}+\int_{\Omega} a(z)|D u|^{q} d z-\int_{\Omega} f(z, u) u d z \text { for all } u \in W_{0}^{1, p}(\Omega) .
$$


Evidently, $k \in C^{1}\left(W_{0}^{1, p}(\Omega)\right)$ and we have

$$
\left\langle k^{\prime}(u), h\right\rangle=p\left\langle A_{p}(u), h\right\rangle+q \int_{\Omega} a(z)|D u|^{q-2}(D u, D h)_{\mathbb{R}^{N}} d z-\int_{\Omega}\left[f_{x}^{\prime}(z, u) u+f(z, u)\right] h d z
$$

for all $h \in W_{0}^{1, p}(\Omega)$.

From Proposition 3.6, we know that

$$
\varphi(\hat{u})=m=\inf \left\{\varphi(u): k(u)=0, u \in W_{0}^{1, p}(\Omega) \backslash\{0\}\right\} .
$$

Then by the Lagrange multiplier rule (see Papageorgiou, Rădulescu and Repovš [25, Theorem 5.5.9]), we can find $\vartheta \geq 0$ such that

$$
\begin{aligned}
& \varphi^{\prime}(\hat{u})+\vartheta k^{\prime}(\hat{u})=0 \text { in } W^{-1, p^{\prime}}(\Omega)=W_{0}^{1, p}(\Omega)^{*}, \\
\Rightarrow \quad & \left\langle\varphi^{\prime}(\hat{u}), \hat{u}\right\rangle+\vartheta\left\langle k^{\prime}(\hat{u}), \hat{u}\right\rangle=0, \\
\Rightarrow \quad & \vartheta\left\langle k^{\prime}(\hat{u}), \hat{u}\right\rangle=0(\text { since } \hat{u} \in \mathcal{N}) .
\end{aligned}
$$

If $\vartheta \neq 0$, then we must have

$$
\begin{gathered}
\left\langle k^{\prime}(\hat{u}), \hat{u}\right\rangle=0 \\
\Rightarrow p\|D \hat{u}\|_{p}^{p}+q \int_{\Omega} a(z)|D \hat{u}|^{q} d z-\int_{\Omega} f(z, \hat{u}) \hat{u} d z=\int_{\Omega} f_{x}^{\prime}(z, \hat{u}) \hat{u} d z \\
\Rightarrow \quad p\left[\|D \hat{u}\|_{p}^{p}+\int_{\Omega} a(z)|D u|^{q} d z-\int_{\Omega} f(z, \hat{u}) \hat{u} d z\right]+(q-p) \int_{\Omega} a(z)|D u|^{q} d z \\
=\int_{\Omega}\left[f_{x}^{\prime}(z, \hat{u}) \hat{u}^{2}-(p-1) f(z, \hat{u}) \hat{u}\right] d z, \\
\Rightarrow \quad 0>(q-p) \int_{\Omega} a(z)|D u|^{p} d z \geq \int_{\Omega}\left[f_{x}^{\prime}(z, \hat{u}) \hat{u}^{2}-(p-1) f(z, \hat{u}) \hat{u}\right] d z \geq 0
\end{gathered}
$$

(recall that $\hat{u} \in \mathcal{N}, q<p$ and see hypotheses $H(a), H(f)(i v)$ ),

a contradiction. Therefore $\vartheta=0$ and so from (28) we have

$$
\begin{aligned}
& \varphi^{\prime}(\hat{u})=0 \text { in } W^{-1, p^{\prime}}(\Omega), \\
\Rightarrow \quad & \hat{u} \in K_{\varphi} \text { and } \hat{u} \text { is a solution of (3). }
\end{aligned}
$$

Invoking Theorem 7.1 of Ladyzhenskaya and Uraltseva [15, p.286] we have

$$
\hat{u} \in W_{0}^{1, p}(\Omega) \cap L^{\infty}(\Omega) .
$$

We claim that $\hat{u} \in \mathcal{N}$ has fixed sign. Arguing indirectly, suppose that $\hat{u}$ is nodal (sign-changing). Then $u^{ \pm} \not \equiv 0$. From (29), we have

$$
\left\langle\varphi^{\prime}(\hat{u}), h\right\rangle=0 \text { for all } h \in W_{0}^{1, p}(\Omega) .
$$

In (30) we first choose $h=\hat{u}^{+} \in W_{0}^{1, p}(\Omega)$ and then $h=-\hat{u}^{-} \in W_{0}^{1, p}(\Omega)$. We obtain

$$
\begin{aligned}
& \left\langle\varphi^{\prime}\left(\hat{u}^{+}\right), \hat{u}^{+}\right\rangle=0 \text { and }\left\langle\varphi^{\prime}\left(-\hat{u}^{-}\right),-\hat{u}^{-}\right\rangle=0, \\
\Rightarrow \quad & \hat{u}^{+} \text {and }-\hat{u}^{-} \in \mathcal{N} .
\end{aligned}
$$

We have

$$
\begin{aligned}
m & =\varphi(\hat{u})=\varphi\left(\hat{u}^{+}\right)+\varphi\left(-\hat{u}^{-}\right) \geq 2 m, \\
\Rightarrow \quad m & =0, \text { a contradiction since } m>0 \text { (see Proposition 3.4). }
\end{aligned}
$$

We conclude that $\hat{u}$ must have fixed sign. 


\section{Nodal solutions}

In this section we produce a nodal solution for problem (3). To this end, we employ the following set, which contains all nodal solutions of (3)

$$
\mathcal{N}_{0}=\left\{y \in W_{0}^{1, p}(\Omega): y^{+} \in \mathcal{N},-y^{-} \in \mathcal{N}\right\} .
$$

Proposition 4.1. If hypotheses $H(a), H(f)$ hold, then $\mathcal{N}_{0} \neq \emptyset$.

Proof. Consider $y \in W_{0}^{1, p}(\Omega)$ such that $y^{+} \not \equiv 0, y^{-} \not \equiv 0$ (that is, a nodal Sobolev function). According to Proposition 3.1, we can find $t_{ \pm}>0$ such that

$$
t_{+} y^{+} \in \mathcal{N} \text { and } t_{-} y^{-} \in \mathcal{N} \text {. }
$$

We set

$$
v=t_{+} y^{+}-t_{-} y^{-}
$$

Evidently, $v^{+}=t_{+} y^{+} \in \mathcal{N}$ and $v^{-}=t_{-} y^{-} \in \mathcal{N}$. Therefore $v \in \mathcal{N}_{0} \neq \emptyset$.

We set

$$
m_{0}=\inf _{\mathcal{N}_{0}} \varphi .
$$

Proposition 4.2. If hypotheses $H(a), H(f)$ hold, then there exists $y_{0} \in \mathcal{N}_{0}$ such that $m_{0}=\varphi\left(y_{0}\right)$.

Proof. Let $\left\{y_{n}\right\}_{n \geq 1} \subseteq \mathcal{N}_{0}$ be a minimizing sequence, that is,

$$
\varphi\left(y_{n}\right) \downarrow m_{0} \text { as } n \rightarrow \infty .
$$

We have

$$
\begin{aligned}
& \varphi\left(y_{n}\right)=\varphi\left(y_{n}^{+}\right)+\varphi\left(-y_{n}^{-}\right) \geq 2 m>0 \text { for all } n \in \mathbb{N} \text { (see Proposition 3.4), } \\
\Rightarrow & m_{0} \geq 2 m>0, \\
\Rightarrow \quad & \left\{\varphi\left(y_{n}^{+}\right)\right\}_{n \geq 1} \text { and }\left\{\varphi\left(-y_{n}^{-}\right)\right\}_{n \geq 1} \text { are bounded. }
\end{aligned}
$$

Then on account of Proposition 3.5, we see that

$$
\left\{y_{n}^{+}\right\}_{n \geq 1} \subseteq W_{0}^{1, p}(\Omega) \text { and }\left\{y_{n}^{-}\right\}_{n \geq 1} \subseteq W_{0}^{1, p}(\Omega) \text { are bounded. }
$$

So, we may assume that

$$
y_{n}^{+} \stackrel{w}{\longrightarrow} v_{1} \text { and } y_{n}^{-} \stackrel{w}{\longrightarrow} v_{2} \text { in } W_{0}^{1, p}(\Omega) .
$$

As in the proof of Proposition 3.6 we show that $v_{1} \neq 0, v_{2} \neq 0$. Invoking Proposition 3.6, we can find $t_{1}>0, t_{2}>0$ such that

$$
t_{1} v_{1} \in \mathcal{N} \text { and } t_{2} v_{2} \in \mathcal{N} \text {. }
$$

We set

$$
y_{0}=t_{1} v_{1}-t_{2} v_{2} \text { with } y_{0}^{+}=t_{1} v_{1}, y_{0}^{-}=t_{2} v_{2} .
$$

We have

$$
\begin{aligned}
m_{0}= & \lim _{n \rightarrow \infty} \varphi\left(y_{n}\right)(\text { see }(31)) \\
= & \lim _{n \rightarrow \infty}\left[\varphi\left(y_{n}^{+}\right)+\varphi\left(-y_{n}^{-}\right)\right] \\
\geq & \liminf _{n \rightarrow \infty}\left[\varphi\left(t_{1} y_{n}^{+}\right)+\varphi\left(-t_{2} y_{n}^{-}\right)\right] \\
& \left(\text {see Proposition } 3.3 \text { and recall that } y_{n}^{+},-y_{n}^{-} \in \mathcal{N}\right) \\
\geq & \varphi\left(t v_{1}\right)+\varphi\left(-t_{2} v_{2}\right) \\
& (\text { from the sequential weak lower semicontinuity of } \varphi) \\
= & \varphi\left(y_{0}\right)(\text { see }(33)) \\
\geq & m_{0}\left(\text { since } y_{0} \in \mathcal{N}_{0}, \text { see }(32),(33)\right), \\
\Rightarrow \varphi\left(y_{0}\right)= & m_{0}, y_{0} \in \mathcal{N}_{0} .
\end{aligned}
$$

The proof is now complete. 
We show that $\mathcal{N}_{0}$ is a natural constraint for $\varphi$. To this end, we will use some tools from Nonsmooth Analysis. In particular, we will use the generalized subdifferential in the sense of Clarke [7]. Let us recall its definition. Suppose that $X$ is a Banach space and $\Psi: X \rightarrow \mathbb{R}$ is a locally Lipschitz function. For every $u, h \in X$, we define

$$
\Psi^{0}(u ; h)=\limsup _{\substack{x \rightarrow u \\ t \downarrow 0}} \frac{\Psi(x+t h)-\Psi(x)}{t} .
$$

Then the mapping $h \mapsto \Psi^{0}(u ; h)$ is continuous and sublinear. We define the set

$$
\partial \Psi(u)=\left\{u^{*} \in X^{*}:\left\langle u^{*}, h\right\rangle_{X} \leq \Psi^{0}(u ; h) \text { for all } h \in X\right\}
$$

with $\langle\cdot, \cdot\rangle_{X}$ being the duality brackets for the pair $\left(X, X^{*}\right)$. By the Hahn-Banach theorem, $\partial \Psi(u) \neq \emptyset$ for all $u \in X$ and is convex and $w^{*}$-compact. The multifunction $u \mapsto \partial \Psi(u)$ is the generalized subdifferential of $\Psi(\cdot)$. This notion has a very rich calculus, extending the smooth calculus and that of Convex Analysis (see Clarke $[7,8])$.

Proposition 4.3. If hypotheses $H(a), H(f)$ hold, then $y_{0} \in K_{\varphi}$ and so $y_{0} \in W_{0}^{1, p}(\Omega) \cap L^{\infty}(\Omega)$ is a nodal solution of problem (3).

Proof. With $k: W_{0}^{1, p}(\Omega) \rightarrow \mathbb{R}$ as in the proof of Proposition 3.7, we have

$$
\varphi\left(y_{0}\right)=m_{0}=\inf \left\{\varphi(y): k\left(y^{+}\right)=0, k\left(-y^{-}\right)=0, y^{ \pm} \neq 0\right\}
$$

(see Proposition 4.2).

In this case we cannot apply the classical multiplier rule since the functions $y \mapsto k_{1}(y)=k\left(y^{+}\right)$and $y \mapsto k_{2}(y)=k\left(-y^{-}\right)$are no longer of class $C^{1}$. However, they are locally Lipschitz and so instead we can use the nonsmooth multiplier rule of Clarke [8, p.221]. So, we can find $\vartheta_{1}, \vartheta_{2} \geq 0$ such that

$$
0 \in \partial\left[\varphi+\vartheta_{1} k_{1}+\vartheta_{2} k_{2}\right]\left(y_{0}\right)
$$

with $\partial\left[\varphi+\vartheta_{1} k_{1}+\vartheta_{2} k_{2}\right]$ being the generalized subdiffrerential in the sense of Clarke of the locally Lipschitz function $u \mapsto \varphi(u)+\vartheta_{1} k_{1}(u)+\vartheta_{2} k_{2}(u)$ defined above. From the sum rule of the subdifferential calculus (see Clarke [8, p.200]) and (34) we have

$$
0=\varphi^{\prime}\left(y_{0}\right)+\vartheta_{1} h_{1}^{*}+\vartheta_{2} h_{2}^{*}
$$

with $h_{1}^{*} \in \partial k_{1}\left(y_{0}\right)$ and $h_{2}^{*} \in \partial k_{2}\left(y_{0}\right)$. On (35) we act with $y_{0}$ and obtain

$$
0=\vartheta_{1}\left\langle h_{1}^{*}, y_{0}^{+}\right\rangle+\vartheta_{2}\left\langle h_{2}^{*},-y_{0}^{-}\right\rangle
$$

(note that $\varphi^{\prime}\left(y_{0}\right)=\varphi^{\prime}\left(y_{0}^{+}\right)+\varphi^{\prime}\left(-y_{0}^{-}\right)$and recall that $\left.y_{0}^{+},-y_{0}^{-} \in \mathcal{N}\right)$.

From the definition of the Clarke generalized subdifferential and the subdifferential calculus (see Clarke [7, pp. 42,76]), we have

$$
\begin{aligned}
& \vartheta_{1}\left\langle h_{1}^{*}, y_{0}^{+}\right\rangle \\
\leq & \vartheta_{1}\left[p\left\|D y_{0}^{+}\right\|_{p}^{p}+q \int_{\Omega} a(z)\left|D y_{0}^{+}\right|^{q} d z-\int_{\Omega}\left[f_{x}^{\prime}\left(z, y_{0}^{+}\right)\left(y_{0}^{+}\right)^{2}+f\left(z, y_{0}^{+}\right) y_{0}^{+}\right] d z\right] \\
= & \vartheta_{1}\left(p\left[\left\|D y_{0}^{+}\right\|_{p}^{p}+\int_{\Omega} a(z)\left|D y_{0}^{+}\right|^{q} d z-\int_{\Omega} f\left(z, y_{0}^{+}\right) y_{0}^{+} d z\right]+(q-p) \int_{\Omega} a(z)\left|D y_{0}^{+}\right| d z\right) \\
- & \vartheta_{1} \int_{\Omega}\left[f_{x}^{\prime}\left(z, y_{0}^{+}\right)\left(y_{0}^{+}\right)^{2}-(p-1) f\left(z, y_{0}^{+}\right) y_{0}^{+}\right] d z \\
\leq & 0\left(\text { since } y_{0}^{+} \in \mathcal{N}, q<p \text { and using hypothesis } H(f)(i v)\right) .
\end{aligned}
$$

Similarly we show that

$$
-\vartheta_{2}\left\langle h_{2}^{*},-y_{0}^{-}\right\rangle \geq 0
$$


From (36), (37) and (38) we infer that

$$
\vartheta_{1}\left\langle h_{1}^{*}, y_{0}^{+}\right\rangle=0 \text { and } \vartheta_{2}\left\langle h_{2}^{*}, y_{0}^{-}\right\rangle=0 .
$$

If $\vartheta_{1} \neq 0$, then $\left\langle h_{1}^{*}, y_{0}^{+}\right\rangle=0$ and since $y_{0}^{+} \in \mathcal{N}$, as in the proof of Proposition 3.7, we have a contradiction. Hence $\vartheta_{1}=0$. Similarly we show that $\vartheta_{2}=0$. Therefore we finally have

$$
\begin{aligned}
& \varphi^{\prime}\left(y_{0}\right)=0(\text { see }(35)), \\
\Rightarrow & y_{0} \in K_{\varphi}, \\
\Rightarrow & y_{0} \in W_{0}^{1, p}(\Omega) \cap L^{\infty}(\Omega) \text { is a nodal solution (3). }
\end{aligned}
$$

The proof is now complete.

So, we can finally state the following multiplicity theorem for problem (3).

Theorem 4.4. If hypotheses $H(a), H(f)$ hold, then problem (3) has a ground state solution $\hat{u} \in W_{0}^{1, p}(\Omega) \cap$ $L^{\infty}(\Omega)$ with fixed sign and a nodal solution $y_{0} \in W_{0}^{1, p}(\Omega) \cap L^{\infty}(\Omega)$.

Acknowledgments. This research was supported by the Slovenian Research Agency grants P1-0292, J1-8131, N1-0064, N1-0083, and N1-0114.

\section{References}

[1] J.M. Ball, Convexity conditions and existence theorems in nonlinear elasticity, Arch. Rational Mech. Anal. 63 (1976/77), no. 4, 337-403.

[2] J.M. Ball, Discontinuous equilibrium solutions and cavitation in nonlinear elasticity, Philos. Trans. Roy. Soc. London Ser. A 306 (1982), no. 1496, 557-611.

[3] P. Baroni, M. Colombo, G. Mingione, Harnack inequalities for double phase functionals, Nonlinear Anal. 121 (2015), 206-222.

[4] P. Baroni, M. Colombo, G. Mingione, Regularity for general functionals with double phase, Calc. Var. Partial Differential Equations 57 (2018), Art 62.

[5] V. Benci, P. D’Avenia, D. Fortunato, L. Pisani, Solitons in several space dimensions: Derrick's problem and infinitely many solutions, Arch. Ration. Mech. Anal. 154 (2000), 297-324.

[6] L. Cherfils, Y. Ilyasov, On the stationary solutions of generalized reaction-diffusion equations with $p$ \& q-Laplacian, Comm. Pure Appl. Anal. 4 (2005), 9-22.

[7] F. Clarke, Optimization and Nonsmooth Analysis, Wiley, New York, 1983.

[8] F. Clarke, Functional Analysis, Calculus of Variations and Optimal Control, Springer-Verlag, London, 2013.

[9] F. Colasuonno, M. Squassina, Eigenvalues for double phase variational integrals, Annali Mat. Pura. Appl. 195 (2016), 1917-1956.

[10] M. Colombo, G. Mingione, Regularity for double phase variational problems, Arch. Ration. Mech. Anal. 215 (2015), 443-496.

[11] M. Colombo, G. Mingione, Bounded minimizers of double phase variational integrals, Arch. Ration. Mech. Anal. 218 (2015), 219-273.

[12] L. Gasinski, N.S. Papageorgiou, Nonlinear Analysis, Chapman \& Hall/CRC, Boca Raton, FL, 2006.

[13] B. Ge, D.J. Lv, J.F. Lu, Multiple solutions for a class of double phase problem without the AmbrosettiRabinowitz conditions, Nonlinear Anal. 188 (2019), 294-315. 
[14] J. Heinonen, T. Kilpeläinen, O. Martio, Nonlinear Potential Theory of Degenerate Elliptic Equations, Clarendon Press, Oxford, 1993.

[15] O. Ladyzhenskaya, N. Uraltseva, Linear and Quasilinear Elliptic Equations, Academic Press, New York, 1968.

[16] G. Lieberman, The natural generalization of the natural conditions of Ladyzhenskaya and Uraltseva for elliptic equations, Comm. Partial Differential Equations 16 (1991), 311-361.

[17] W. Liu, G. Dai, Existence and multiplicity results for double phase problems, J. Differential Equations 265 (2018), 4311-4334.

[18] P. Marcellini, On the definition and the lower semicontinuity of certain quasiconvex integrals, Ann. Inst. H. Poincaré, Anal. Non Linéaire 3 (1986), 391-409.

[19] P. Marcellini, Regularity and existence of solutions of elliptic equations with $p, q$-growth conditions, $J$. Differential Equations 90 (1991), 1-30.

[20] P. Marcellini, Everywhere regularity for a class of elliptic systems without growth conditions, Ann. Scuola Norm. Sup. Pisa Cl. Sci. (4) 23 (1996), no. 1, 1-25.

[21] Ch.B. Morrey, Jr., Multiple Integrals in the Calculus of Variations, Reprint of the 1966 edition. Classics in Mathematics, Springer-Verlag, Berlin, 2008.

[22] D. Mugnai, N.S. Papageorgiou, Wang's multiplicity result for superlinear $(p, q)$-equations without the Ambrosetti-Rabinowitz condition, Trans. Amer. Math. Soc. 366 (2014), 4919-4937.

[23] N.S. Papageorgiou, V.D. Rădulescu, Nonlinear nonhomogeneous Robin problems with superlinear reaction, Adv. Nonlin. Studies 16 (2016), 737-764.

[24] N.S. Papageorgiou, V.D. Rădulescu, D.D. Repovš, Double-phase problems with reaction of arbitrary growth, Z. Angew. Math. Phys. 69 (2018), no. 4, Art. 108, 21 pp.

[25] N.S. Papageorgiou, V.D. Rădulescu, D.D. Repovš, Nonlinear Analysis - Theory and Methods, Springer Monographs in Mathematics, Springer Nature, Cham, 2019.

[26] N.S. Papageorgiou, V.D. Rădulescu, D.D. Repovš,, Double-phase problems and a discontinuity property of the spectrum, Proc. Amer. Math. Soc. 147 (2019), no. 7, 2899-2910.

[27] N.S. Papageorgiou, F. Vetro, C. Vetro, A multiplicity theorem for parametric superlinear $(p, q)$ equations, Opuscula Math., to appear.

[28] N.S. Papageorgiou, P. Winkert, Applied Nonlinear Functional Analysis, Walter de Gruyter, Berlin, 2018.

[29] P. Pucci, J. Serrin, The Maximum Principle, Birkhäuser, Basel, 2007.

[30] Z. Qihu, V.D. Rădulescu, Double phase anisotropic variational problems and combined effects of reaction and absorption terms, J. Math. Pures Appl. 118 (2018), 159-203.

[31] V. D. Rădulescu, Isotropic and anisotropic double phase problems: old and new, Opuscula Math. 39 (2019), 259-279.

[32] V.V. Zhikov, Averaging of functionals of the calculus of variations and elasticity, Math. USSR-Izves. 29 (1987), 33-66.

[33] V.V. Zhikov, On variational problems and nonlinear elliptic equations with nonstandard growth conditions, J. Math. Sci. 173 (2011), 463-570. 